\title{
Investigation and Analysis of the Status Quo of Prevention and Control Practices of Catheter-Line Associated Bloodstream Infections (CLABSI) in Guangxi, China
}

\author{
Caijiao Wu ${ }^{1 *}$, Huihan Zhao ${ }^{*}$, Galal A. Al-Samhari'2, Qingjuan Jiang1, \\ Ying Ling1\#, Yanping Ying" ${ }^{1 \#}$ \\ ${ }^{1}$ Department of Nursing, The First Affiliated Hospital of Guangxi Medical University, Nanning, China \\ ${ }^{2}$ Department of Epidemiology and Biostatistics School of Public Health, Guangxi Medical University, Nanning, China \\ Email: 1625712125@qq.com, zhaohuihan2@sina.com, alsamhary2009@tu.edu.ye,1061315095@qq.com, \\ "lingying1983@126.com, "yanpingying0116@126.com
}

How to cite this paper: $\mathrm{Wu}, \mathrm{C} . J .$, Zhao, H.H., Al-Samhari, G.A., Jiang, Q.J., Ling, Y. and Ying, Y.P. (2021) Investigation and Analysis of the Status Quo of Prevention and Control Practices of Catheter-Line Associated Bloodstream Infections (CLABSI) in Guangxi, China. Advances in Infectious Diseases, 11, 333-343.

https://doi.org/10.4236/aid.2021.114030

Received: September 25, 2021

Accepted: October 25, 2021

Published: October 28, 2021

Copyright $\odot 2021$ by author(s) and Scientific Research Publishing Inc. This work is licensed under the Creative Commons Attribution International License (CC BY 4.0).

http://creativecommons.org/licenses/by/4.0/

\begin{abstract}
The prevention and control of catheter line-associated bloodstream infections (CLABSI) have become the key content of medical care and have become the core improvement goal of nursing quality control. However, the prevention and control status of CLABSI in hospitals of different grades in Guangxi is not clear. In this study, we aim to investigate central venous catheter (CVC) placement and disinfectant use in second and third-level hospitals in Guangxi. This survey was conducted on the second and third-level hospitals in Guangxi, China from 13th April 2021 to 19th April 2021. The results show that a total of 283 questionnaires were collected, including 206 secondary hospitals and 77 tertiary hospitals. In terms of the CVC, tertiary hospitals were able to place CVC entirely under the guidance of B-ultrasound, which was $24(31.6 \%)$ and secondary hospitals were 26 (20.6\%). In secondary hospitals, Most CVC placements were performed in operating rooms 94 (74.6\%) and $65(85.5 \%)$ on the third level hospital, but $32.5 \%$ of secondary hospitals and $48.7 \%$ of tertiary hospitals were selected at the bedside of patients in general wards, and $27.8 \%$ of the second-level hospital, $43.4 \%$ of third-level hospitals was done in general ward treatment rooms, only $61.9 \%$ of secondary hospitals and $64.5 \%$ of tertiary hospitals could fully achieve the maximum sterile barrier. In terms of skin disinfectants, only $36.0 \%$ of tertiary hospitals and $16.4 \%$ of second-level CVC-operators chose $>0.5 \%$ chlorhexidine alcohol. In conclusion, the prevention and control of catheter line-associated
\end{abstract}

*The first authors

\#Corresponding authors. 
bloodstream infections (CLABSI) in Guangxi are not ideal. The prevention and control department should increase training, implement guidelines and standardize management to reduce the incidence of CLABSI.

\section{Keywords}

Central Venous Catheter, Catheter-Line Associated Bloodstream Infections, Prevention, Quality Control, Survey

\section{Introduction}

CVC refers to the catheters that enter the jugular vein, subclavian vein, or femoral vein through skin puncture, and their head ends are located in the proximal superior vena cava, right atrium and inferior vena cava [1]. The common types of CVC are tunnel CVC, non-tunnel CVC, and Port [2]. In daily medical practice, CVC is mainly used for infusion, blood transfusion, infusion stimulants, and hemodynamic monitoring in daily medical practice and has become an indispensable means of treatment and rescue. However, their complications are becoming increasingly prominent, among which Central line-associated bloodstream infections (CLABSI) is one of the most serious complications [3] [4] [5]. There are two ways to describe CVC-related bloodstream infection: catheter-related bloodstream infection (CRBSI), central line-associated bloodstream infections (CLABSI), the most commonly used is CLABSI [4] [6]. CLABSI aggravates patients' pain and prolongs the length of hospital stay, increases the economic burden of patients, wastes medical resources, and may endanger the lives of patients in serious cases [7] [8] [9]. According to statistics, about 250,000 cases of CLABSI in the United States every year, about 90\% occur in CVC [10] [11]. However, the incidence of CLABSI in developing countries is five times higher than that in the United States, and the rate of CLABSI in China is 2.3 per 1000 catheter-days (786 events in 344,758 catheter-days) [12] [13]. It has been reported that about $65 \%-70 \%$ of CLABSI can be prevented every year [14]. CVC placement and maintenance are two important reasons for CLABSI [15]. However, the extent to which the secondary and tertiary hospitals used CLABSI prevention measures is still unknown in Guangxi. Therefore, a self-made questionnaire was used to investigate CVC placement and the use of disinfectants in medical institutions above the second level in Guangxi to provide a reference for promoting the prevention and control strategy of CLABSI and reducing the incidence of CLABSI.

\section{Methods}

Investigate the second and third-level medical institutions in Guangxi, China. Hospital inclusion criteria were in line with the second and third-level hospital standards specified in the "Hospital Grade Evaluation Standards". The questionnaire was conducted according to the related contents of the guideline for 
preventing and controlling vascular catheter-related infection issued by the National Health Commission [16] and related literature. It was also discussed by experts in related fields of intravenous therapy and Nursing Quality Control Center members. The first draft of the CLABSI Prevention and Control Status Questionnaire was formed. The questionnaire has undergone two rounds of pre-investigation and revised the entries that are questionable, unclear, or difficult to fill in, and the final version of the CLABSI Prevention and Control Status Questionnaire was done. The questionnaire mainly includes two parts; the first part is general hospital information, which mainly includes the basic information of the hospital level, the second one is the current status of CLABSI prevention and control. The specific investigation content includes catheter development, whether the CVC is implanted under the guidance of B-ultrasound, the implantation environment, the most significant sterile barrier, and the skin disinfectant used. The overall Cronbach's $\alpha$ coefficient of the questionnaire is 0.960 , and the construct validity is 0.961 .

Use the questionnaire star platform to issue online questionnaires, which will be issued and filled out from 13th April 2021 to 19th April 2021, for a total of seven days. The CLABSI Prevention and Control Status Questionnaire is carefully filled and issued by the nursing quality control managers and submitted, collecting 283 questionnaires.

Excel (Microsoft Office 2016) and Statistical Package for 6 Social Sciences (SPSS, Version 21.0) were used to analyze the data. The enumeration data were described by the rate (\%), and the chi-square test was used for statistical inference, and the difference was statistically significant with $\mathrm{P}<0.05$.

\section{Results}

A total of 283 copies of the CLABSI Prevention and Control Status Questionnaire were collected, of which 77 are tertiary hospitals (27.2\%) and there are 206 secondary hospitals (72.8\%) (Figure 1).

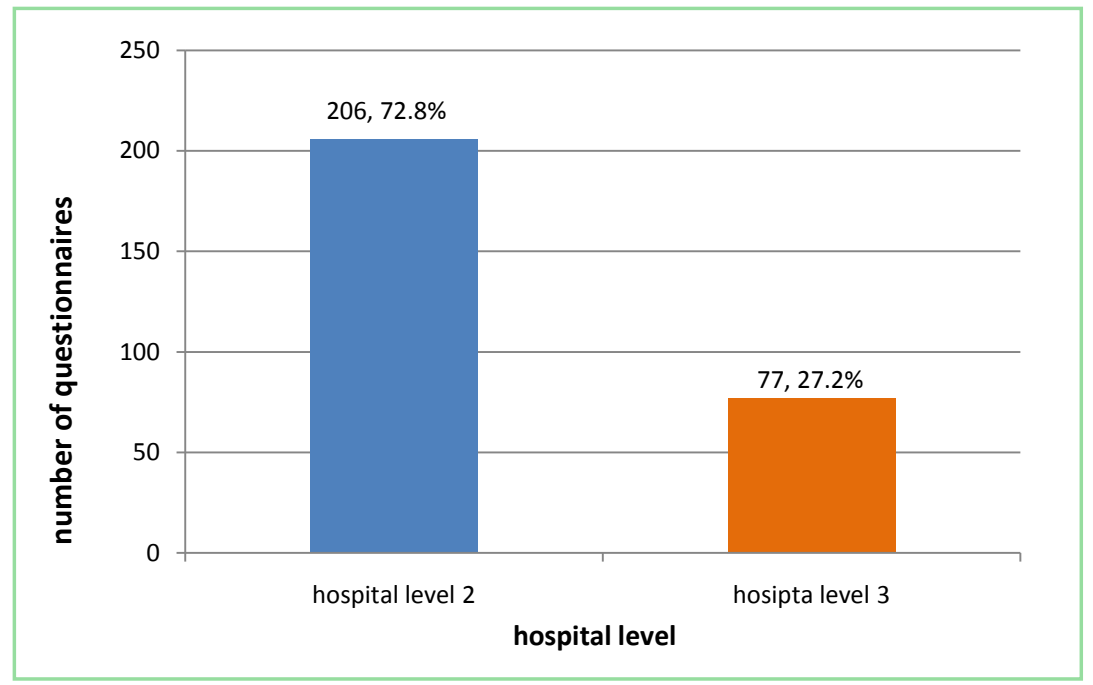

Figure 1. Basic information among 383 hospitals surveyed. 
The survey results show that almost all (98.7\%) third-level hospitals have implemented CVC intubation technology, $61.2 \%$ of second-level hospitals have implemented CVC technology, and $38.8 \%$ of hospitals have not implemented CVC technology. Only $31.6 \%$ of third-level hospitals can fully perform CVC catheterization under the guidance of B-ultrasound, and $25.0 \%$ of tertiary hospitals cannot achieve CVC catheterization under the guidance of B-ultrasound at all. Only $20.6 \%$ of second-level hospitals can fully perform CVC catheterization under the guidance of B ultrasound. $42.1 \%$ of CVC placement under super guidance was utterly impossible. CVC implantation in third-level hospitals is mainly performed in the operating room (85.5\%) and intensive care unit (84.2\%) environment, and CVC implantation in second-level hospitals is also mostly performed in the operating room (74.6\%) and intensive care unit (61.9\%) environment. However, the CVC implantation part of the third-level hospitals is carried out in the bedside $(48.7 \%)$ of the general ward and the general ward's treatment room (43.4\%). At the same time, the CVC implantation in the second-level hospital chooses to be at the bedside of the general ward (32.5\%), and the treatment room $(27.8 \%)$ of the general ward. Our survey data shows that $61.9 \%$ and $64.5 \%$ of the second-and third-level hospitals can fully achieve the maximum sterile barrier when CVC is placed. $38.1 \%$ of secondary versus $32.9 \%$ of tertiary hospitals can partially use the maximal sterile barrier. Only $2.6 \%$ of tertiary hospitals cannot use maximal sterile-barrier precautions before CVC insertion (Table 1).

Table 1. CVC placement in hospitals of different grades.

\begin{tabular}{|c|c|c|c|}
\hline \multirow{2}{*}{ Variables } & \multicolumn{2}{|c|}{$\mathrm{CVC}$} & \multirow{2}{*}{ P-value } \\
\hline & Hospital level 2 & Hospital level 3 & \\
\hline \multicolumn{4}{|l|}{ CVC Development } \\
\hline Yes & $126(61.2 \%)$ & $76(98.7 \%)$ & \multirow{2}{*}{0.000} \\
\hline No & $80(38.8 \%)$ & $1(1.3 \%)$ & \\
\hline \multicolumn{4}{|l|}{ Ultrasound-guided catheterization } \\
\hline Completely & $26(20.6 \%)$ & $24(31.6 \%)$ & \multirow{3}{*}{0.037} \\
\hline Partially & $47(37.3 \%)$ & $33(43.4 \%)$ & \\
\hline No & $53(42.1 \%)$ & $19(25.0 \%)$ & \\
\hline \multicolumn{4}{|l|}{ Catheterization environment } \\
\hline Bedside of patients in the general ward & $41(32.5 \%)$ & $37(48.7 \%)$ & 0.022 \\
\hline The general ward treatment room & $35(27.8 \%)$ & $33(43.4 \%)$ & 0.023 \\
\hline Operation room & $94(74.6 \%)$ & $65(85.5 \%)$ & 0.066 \\
\hline ICU & $78(61.9 \%)$ & $64(84.2 \%)$ & 0.001 \\
\hline Others & $7(5.6 \%)$ & $6(7.9 \%)$ & 0.512 \\
\hline \multicolumn{4}{|l|}{ Maximal sterile barrier } \\
\hline Completely & $78(61.9 \%)$ & $49(64.5 \%)$ & \\
\hline Partially & $48(38.1 \%)$ & $25(32.9 \%)$ & 0.156 \\
\hline No & $0(0.0 \%)$ & $2(2.6 \%)$ & \\
\hline
\end{tabular}

${ }^{\star} \mathrm{CVC}$-central venous catheter. 
The survey founded that: before CVC puncture, a higher percentage of the secondary versus tertiary hospitals $(78.4 \%$ versus $81.3 \%)$ reported choosing $>0.5$ iodophors for the skin preparation. $66.4 \%$ of secondary versus $61.3 \%$ tertiary hospitals chose $75 \%$ alcohol for skin disinfection. However, only $36.0 \%$ of tertiary hospitals used more than $0.5 \%$ chlorhexidine for skin disinfection around CVC. At the same time, only $16.4 \%$ of the secondary hospitals used more than $0.5 \%$ chlorhexidine. There is no difference between the hospital levels $\left(\chi^{2}=\right.$ $1.500, \mathrm{P}=0.221$ ) (Figure 2).

\section{Discussion}

B-guided CVC placement needs to be improved. B-guided CVC implantation has many advantages, reducing the pain caused by repeated punctures of the patient. At the same time, if the blind puncture cannot be successfully punctured at one time, it will increase the frequency of repeated catheter insertion and adjustment and prolong the time of contact with the external environment. B-ultrasound guidance can increase the success rate of puncture, shorten the catheterization time, and improve the quality and safety of central venous catheterization, thereby reducing the incidence of CLABSI [4] [17] [18] [19]. In this survey, it was found that only $50(24.8 \%)$ medical institutions can insert CVC entirely under the guidance of B-ultrasound, and only 80 (39.6\%) hospitals can insert CVC under the guidance of partial B-ultrasound. At the same time, 72 (35.6\%) medical institutions cannot insert CVC under the guidance of B-ultrasound. Moreover, there are differences in different levels of hospitals, which may be related to the

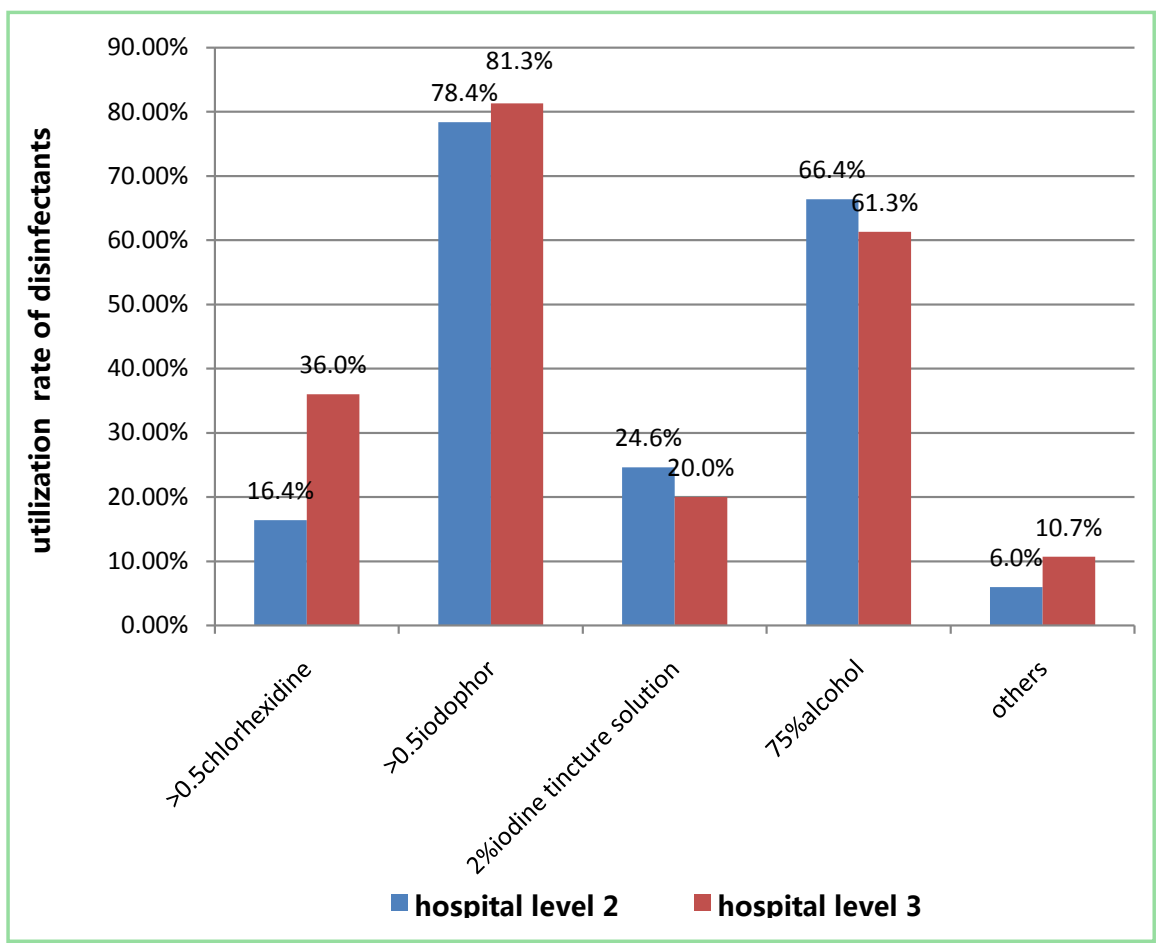

Figure 2. The usage rate of skin disinfectants for CVC implantation sites in different levels of medical institutions. 
limitations of CVC implantation technology in the development of second-level hospitals, and related technologies and operations are not mature enough. A randomized controlled study found that compared with the control group given conventional catheterization, the incidence of catheter bloodstream infection in the experimental group guided by B ultrasound was significantly reduced (the infection rate of the control group was $6.15 \%$, the experimental group is $0.77 \%$ ), and it is statistically significant. Therefore, B-ultrasound-guided placement of CVC can reduce the incidence of CLABSI [20]. Another prospective randomized controlled study also found that compared with the traditional method of CVC placement, ultrasound-guided placement of CVC not only increased the puncture success rate $(93.9 \%$ vs. $78.5 \%)$, but also reduced the incidence of complications ( $4.6 \%$ vs. $16.9 \%$ ), and the $\mathrm{P}$ values were all $<0.05$ [21]. A recent randomized study found that ultrasound-guided internal jugular vein puncture has a significantly lower incidence of CLABSI compared with "blind" venous puncture. This may be relevant with ultrasound-guided placement of CVC that can reduce tissue damage and shorten surgery time [22]. The National Health Commission issued the "Guidelines for the Prevention and Control of Vascular Catheter-Related Infections" (2021 version), which pointed out that if CVC catheterization is performed for patients with poor vascular conditions, ultrasound-guided puncture can be used in hospitals where conditions permit. Therefore, it is recommended that medical institutions can use ultrasound guidance to insert CVC and pay attention to aseptic operation when using high-frequency linear array probes.

There is a gap between the CVC tube environment and the guidelines. This survey shows that most CVC implements in the third and second-class hospitals are carried out in the operating room and intensive care room, but some hospitals applied it bedside the patients in the general ward (third-level hospitals: 48.7\%, second-level hospitals Hospital: 32.5\%). Meanwhile, 43.4\% of the third-level hospitals and $27.8 \%$ of the second hospital chose to put CVC into the treatment room of the general ward. The guideline for prevention and control of vascular catheter-related infection (2021 Edition) [16] pointed out that the central catheter placement environment should meet the requirements of class II environment of Sanitary standard for hospital disinfection. However, bedside the patients and treatment room of general wards, the environmental catheterization cannot meet the requirements of the class II environment, so improving the operating environment is an urgent problem. The operating environment is an influential factor of CLABSI and may interfere with the maximum aseptic barrier. In a foreign randomized controlled experiment (CRT), patients who were placed in the operating room were randomly divided into two groups: maximal sterile barrier and the standard aseptic barrier. The results showed no difference between the two groups in preventing catheter-related blood infection, and the maximum aseptic barrier group could not reduce the incidence of CLABSI [23]. The effect of the most significant aseptic barrier may also be related to the environment of CVC implantation. However, we still need a prospective, large sample, multicenter experimental research to confirm this conclusion. Although 
there is no reliable evidence to support it, it is recommended to place the CVC in the operating room and intensive care room if possible.

The implementation of the maximal sterile barrier is still insufficient. The maximal sterile barrier refers to implementing the greatest aseptic barrier in order to prevent CLABSI before the catheterization process; the operator and assistant are required to carry out hand hygiene, wear a hat, mask and sterile gloves, wear aseptic clothes, the hat should cover all hair, and the mask should cover the nose and mouth according to the relevant requirements of "hand hygiene standard for medical personnel". It is required that the large sterile drape covers whole the body and only exposes the puncture site for the patient [16] [23]. The survey data showed that only $61.9 \%$ and $64.5 \%$ of the secondary and tertiary hospitals achieved the maximum aseptic barrier, and some hospitals did not achieve the maximum aseptic barrier. Therefore, whether it is a secondary hospital or a tertiary hospital, the quality control department of the hospital should pay attention to applying the maximum aseptic barrier in the process of catheterization and the clinical implementation rate urgently needs to be improved. Several guidelines in China and outside China clearly pointed out that the maximum aseptic barrier should be used to cover patients' whole body, whether it is a CVC or arterial catheter [15] [16] [24] [25]. A prospective randomized controlled study was done in 82 patients with CVC implantation; they conducted that under the premise of using the same gauze dressing, the positive rate of bacterial colonization was $41 \%$ in the group with the largest sterile barrier and $60 \%$ in the group with the lowest sterile barrier [26]. Therefore, the maximum sterile barrier can reduce the risk of bacterial infection of the skin at the catheter site. A prospective study has done on 176 patients that catheterized under maximal sterile barrier precautions were comparable to the 167 control group that only used sterile gloves and small drapes. Only four cases of catheter-related infection occurred in the experimental group, while 12 cases occurred in the control group; for that, the maximum aseptic barrier measure can reduce the incidence of CLABSI and increase cost-effectiveness [27]. Therefore, in the absence of more reliable clinical evidence, it is still recommended to select the maximum sterile barrier as a preventive measure in the daily process of CVC insertion to reduce the incidence of catheter-related bloodstream infection as much as possible.

The selection of disinfectants is not standardized. The bacteria on the skin surface of the puncture site are the primary source for the infection when the CVC is placed [28]. Therefore, it is important to select an effective disinfectant before the catheter placement. This survey found that most of the secondary and tertiary hospitals mainly used iodophor and $75 \%$ alcohol for skin disinfection in CVC placement. Only $36.0 \%$ of tertiary hospitals chose more than $0.5 \%$ of the chlorhexidine alcohol concentration for skin disinfection around the CVC, and only $16.4 \%$ of the second-level hospitals, A similar result founded by investigating prevention and control of Catheter-Related Blood Stream Infections in 122 hospitals in China [29]. The current use of skin disinfectants is not standardized, 
so hospitals at all levels need to regulate the selection of disinfectants to prevent and control the occurrence of CLABSI. When the CVC is placed, the choice of chlorhexidine disinfectant can not only reduce the incidence of CLABSI (1.60\%) and mortality $(0.23 \%)$, but also reduce the economic burden of patients [30]. Meanwhile, studies have found that the use of $2 \%$ chlorhexidine gluconate disinfectant can effectively prevent and control the occurrence of blood infection related to CVC, and the risk of infection can be reduced by $49 \%$, which is considered to be a safe, economical and effective skin disinfectant, and it is worth to be used in the clinical department [31]. The domestic guidelines for preventing and controlling vascular catheter-related infection suggest that chlorhexidinealcohol $>0.5 \%$ disinfectant should be used as the first choice of skin disinfectant indwelling catheter. However, most medical institutions fail to carry out clinical practice in accordance with the relevant guidelines for CLABSI prevention and control. The possible reasons are that nursing management personnel and relevant technical operators have knowledge shortage in this field, and may also be related to the relevant consumables purchase management department of medical institutions, so many measures are needed to promote clinical operators and relevant management departments to carry out evidence-based clinical practice and management.

The limitation of this study is that it only involved Guangxi, and did not investigate the prevention and control status of CLABSI in other provinces.

\section{Conclusion}

There is still a gap between the implementation of CLABSI prevention and control in Guangxi and the relevant guidelines and industry standards, and there are some differences among different hospital levels. Therefore, clinical quality control monitoring departments need to target the vital issues and gradually promote the implementation and also the standardized management of CLABSI prevention and control work to reduce the incidence of catheter-related bloodstream infections.

\section{Acknowledgements}

The authors wish to thank Nursing Quality Control Center members for their invaluable contributions to this investigation.

\section{Funding Sources}

This work was supported by The National Natural Science Foundation of China [grant number 81860032], and the First Affiliated Hospital of Guangxi Medical University Nursing Clinical Research Climbing Project, Category A Peak Project [grant number YYZS2020025].

\section{Conflicts of Interest}

The authors declare no conflicts of interest regarding the publication of this paper. 


\section{References}

[1] Lockwood, J. and Desai, N. (2019) Central Venous Access. British Journal of Hospital Medicine, 80, c114-c119. https://doi.org/10.12968/hmed.2019.80.8.C114

[2] Johansson, E., Hammarskjöld, F., Lundberg, D., et al. (2013) Advantages and Disadvantages of Peripherally Inserted Central Venous Catheters (PICC) Compared to Other Central Venous Lines: A Systematic Review of the Literature. Acta Oncologica, 52, 886-892. https://doi.org/10.3109/0284186X.2013.773072

[3] Lai, N.M., Lai, N.A., O’Riordan, E., et al. (2016) Skin Antisepsis for Reducing Central Venous Catheter-Related Infections. Cochrane Database of Systematic Reviews, 7, Cd010140. https://doi.org/10.1002/14651858.CD010140.pub2

[4] Bell, T. and O'Grady, N.P. (2017) Prevention of Central Line-Associated Bloodstream Infections. Infectious Disease Clinics of North America, 31, 551-559. https://doi.org/10.1016/j.idc.2017.05.007

[5] Lutwick, L., Al-Maani, A.S., Mehtar, S., et al. (2019) Managing and Preventing Vascular Catheter Infections: A Position Paper of the International Society for Infectious Diseases. International Journal of Infectious Diseases, 84, 22-29. https://doi.org/10.1016/j.ijid.2019.04.014

[6] Seo, H.K., Hwang, J.H., Shin, M.J., et al. (2018) Two-Year Hospital-Wide Surveillance of Central Line-Associated Bloodstream Infections in a Korean Hospital. Journal of Korean Medical Science, 33, e280. https://doi.org/10.3346/jkms.2018.33.e280

[7] Jeong, I.S., Park, S.M., Lee, J.M., et al. (2013) Effect of Central Line Bundle on Central Line-Associated Bloodstream Infections in Intensive Care Units. American Journal of Infection Control, 41, 710-716. https://doi.org/10.1016/j.ajic.2012.10.010

[8] Ziegler, M.J., Pellegrini, D.C. and Safdar, N. (2015) Attributable Mortality of Central Line Associated Bloodstream Infection: Systematic Review and Meta-Analysis. Infection, 43, 29-36. https://doi.org/10.1007/s15010-014-0689-y

[9] Aloush, S.M. and Alsaraireh, F.A. (2018) Nurses' Compliance with Central Line Associated Blood Stream Infection Prevention Guidelines. Saudi Medical Journal, 39, 273-279. https://doi.org/10.15537/smj.2018.3.21497

[10] Raad, I., Hanna, H. and Maki, D. (2007) Intravascular Catheter-Related Infections: Advances in Diagnosis, Prevention, and Management. The Lancet Infectious Diseases, 7, 645-657. https://doi.org/10.1016/S1473-3099(07)70235-9

[11] Ozakin, E., Can, R., Acar, N., et al. (2014) An Evaluation of Complications in Ultrasound-Guided Central Venous Catheter Insertion in the Emergency Department. Turkish Journal of Emergency Medicine, 14, 53-58. https://doi.org/10.5505/1304.7361.2014.93275

[12] Zhong, Q., Hu, B.J., Gao, X.D., et al. (2011) Targeted Surveillance of Catheter-Related Infections in ICU of 65 Hospitals in Shanghai during 2009-2010. Chinese Journal of Hospital Infection, 21, 2408-2410.

[13] Rosenthal, V.D., Al-Abdely, H.M., El-Kholy, A.A., et al. (2016) International Nosocomial Infection Control Consortium Report, Data Summary of 50 Countries for 2010-2015: Device-Associated Module. American Journal of Infection Control, 44, 1495-1504. https://doi.org/10.1016/j.ajic.2016.08.007

[14] Umscheid, C.A., Mitchell, M.D., Doshi, J.A., et al. (2011) Estimating the Proportion of Healthcare-Associated Infections That Are Reasonably Preventable and the Related Mortality and Costs. Infection Control \& Hospital Epidemiology, 32, 101-114. https://doi.org/10.1086/657912 
[15] Ling, M.L., Apisarnthanarak, A., Jaggi, N., et al. (2016) APSIC Guide for Prevention of Central Line Associated Bloodstream Infections (CLABSI). Antimicrobial Resistance \& Infection Control, 5, 16. https://doi.org/10.1186/s13756-016-0116-5

[16] (2021) Guidelines for Prevention and Control of Vessel Catheter Associated Infection (2021 Edition). Chinese Journal of Infection Control, 20, 387-388.

[17] Bodenham, A. (2020) Ultrasound-Guided Vascular Access. European Journal of Anaesthesiology, 37, 341-343. https://doi.org/10.1097/EJA.0000000000001174

[18] Chew, S.C., Beh, Z.Y., Hakumat Rai, V.R., et al. (2020) Ultrasound-Guided Central Venous Vascular Access-Novel Needle Navigation Technology Compared with Conventional Method: A Randomized Study. The Journal of Vascular Access, 21, 26-32. https://doi.org/10.1177/1129729819852057

[19] Leibowitz, A., Oren-Grinberg, A. and Matyal, R. (2020) Ultrasound Guidance for Central Venous Access: Current Evidence and Clinical Recommendations. Journal of Intensive Care Medicine, 35, 303-321. https://doi.org/10.1177/0885066619868164

[20] Huang, W.D., Jiang, F. and Mo, M.J. (2015) Impact of Ultrasound-Guided Subclavian Vein Catheterization on Central Venous Catheter-Related Bloodstream Infections. Chinese Journal of Infection Control, 25, 4920-4921+4928.

[21] Leung, J., Duffy, M. and Finckh, A. (2006) Real-Time Ultrasonographically-Guided Internal Jugular Vein Catheterization in the Emergency Department Increases Success Rates and Reduces Complications: A Randomized, Prospective Study. Annals of Emergency Medicine, 48, 540-547.

https://doi.org/10.1016/j.annemergmed.2006.01.011

[22] Karakitsos, D., Labropoulos, N., De Groot, E., et al. (2006) Real-Time Ultrasound-Guided Catheterisation of the Internal Jugular Vein: A Prospective Comparison with the Landmark Technique in Critical Care Patients. Critical Care, 10, R162. https://doi.org/10.1186/cc5101

[23] Ishikawa, Y., Kiyama, T., Haga, Y., et al. (2010) Maximal Sterile Barrier Precautions Do Not Reduce Catheter-Related Bloodstream Infections in General Surgery Units: A Multi-Institutional Randomized Controlled Trial. Annals of Surgery, 251, 620-623. https://doi.org/10.1097/SLA.0b013e3181d48a6a

[24] O'Grady, N.P., Alexander, M., Burns, L.A., et al. (2011) Guidelines for the Prevention of Intravascular Catheter-Related Infections. American Journal of Infection Control, 39, S1-S34. https://doi.org/10.1016/j.ajic.2011.01.003

[25] Device, T.E.G.o.S.M.o.C.V.A. (2020) The Expert Consensus on Safety Management of Central Venous Access Device. Chinese Journal of Surgery, 58, 261-272.

[26] Carrer, S., Bocchi, A., Bortolotti, M., et al. (2005) Effect of Different Sterile Barrier Precautions and Central Venous Catheter Dressing on the Skin Colonization around the Insertion Site. Minerva Anestesiologica, 71, 197-206.

[27] Raad, I.I., Hohn, D.C., Gilbreath, B.J., et al. (1994) Prevention of Central Venous Catheter-Related Infections by Using Maximal Sterile Barrier Precautions during Insertion. Infection Control \& Hospital Epidemiology, 15, 231-238. https://doi.org/10.1086/646902

[28] Mimoz, O., Lucet, J.C., Kerforne, T., et al. (2015) Skin Antisepsis with Chlorhexidine-Alcohol Versus Povidone Iodine-Alcohol, with and without Skin Scrubbing, for Prevention of Intravascular-Catheter-Related Infection (Clean): An Open-Label, Multicentre, Randomised, Controlled, Two-by-Two Factorial Trial. The Lancet, 386, 2069-2077. https://doi.org/10.1016/S0140-6736(15)00244-5

[29] Zhong, S., Li, H., Guomin, Z., et al. (2017) A Survey of Status of How Nurses Carry 
Out Clinical Practice of Prevention and Control of Catheter-Related Blood Stream Infections. Chinese Nursing Management, 17, 1530-1535.

[30] Chaiyakunapruk, N., Veenstra, D.L., Lipsky, B.A., et al. (2003) Vascular Catheter Site Care: The Clinical and Economic Benefits of Chlorhexidine Gluconate Compared with Povidone Iodine. Clinical Infectious Diseases, 37, 764-771. https://doi.org/10.1086/377265

[31] Chaiyakunapruk, N., Veenstra, D.L., Lipsky, B.A., et al. (2002) Chlorhexidine Compared with Povidone-Iodine Solution for Vascular Catheter-Site Care: A Meta-Analysis. Annals of Internal Medicine, 136, 792-801.

https://doi.org/10.7326/0003-4819-136-11-200206040-00007 